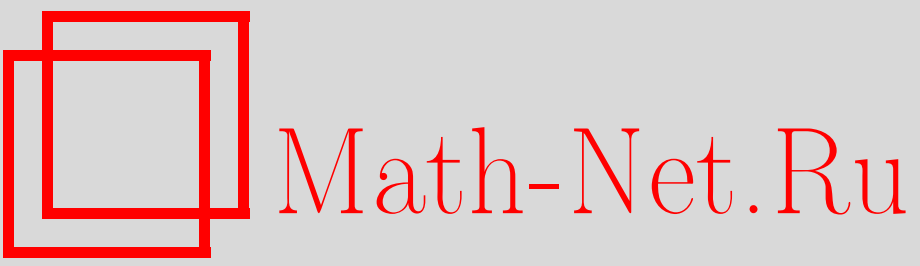

Джин Вен, Эргодичность систем частиц, ТМФ, 2002, том 131, номер 3, 419-431

DOI: https://doi.org/10.4213/tmf338

Использование Общероссийского математического портала Math-Net.Ru подразумевает, что вы прочитали и согласны с пользовательским соглашением

http://www.mathnet.ru/rus/agreement

Параметры загрузки:

IP : 54.80 .73 .141

26 апреля 2023 г., 09:42:37 
ТЕОРЕТИЧЕСКАЯ

И МАТЕМАТИЧЕСКАЯ

ФИЗИКА

Том 131, № 3

июнь, 2002

(C) 2002 г

\section{Джин Вен Чен ${ }^{*}$ \\ ЭРГОДИЧНОСТЬ СИСТЕМ ЧАСТИЦ}

Исследуются эргодичность относительно сдвигов, перемешивание и связанные с этим проблемы инвариантных мер для систем взаимодействующих частиц, таких как ферромагнитные стохастические модели Изинга, процессы контактов, системы с исключением, системы избирателей с тремя возможными стохастически предвзятыми мнениями или со многими возможными мнениями и т.п. Полученные результаты дают ответы на некоторые вопросы, касающиеся этих моделей. В основе используемых методов лежит дуальность.

\section{1. ВВЕДЕНИЕ}

Одной из важнейших проблем, относяшихся к системам взаимодействуюших частиш, является вопрос об их эргодичности, который решается путем изучения инвариантных мер. Если рассматриваемая система инвариантна относительно некоторых сдвигов, то проблема может быть сведена к исследованию мер, инвариантных относительно этих сдвигов. Эргодичность таких инвариантных мер представляет особый интерес. В частности, если система имеет единственную инвариантную меру, то возникает вопрос, является ли она эргодической относительно сдвигов. Ответ на этот вопрос был неизвестен для систем переориентируюшихся спинов (см. [1], гл. III). Он был найден Анджелом в работе [2], где также исследовались более обшие вопросы, касаюшиеся перемешивания и эргодичности относительно сдвигов. Но для большинства детально исследованных неэргодических систем, таких как системы избирателей, процессы контактов, системы с исключением и т.п., ответов на эти вопросы найдено не было. Цель данной работы предложить метод исследования таких проблем. Полученные результаты применимы как ко всем вышеупомянутым системам, так и к широкому классу других интересных моделей. Наши рассуждения основаны на дуальности, которая позволяет свести исследуемые проблемы к другим, относяшимся к более простым системам.

Приведем точные описания моделей и формулировки проблем. Рассматриваемые нами системы взаимодействуюших частиц описываются марковскими процессами с непрерывным временем и пространством состояний $E=W^{Z^{d}}$, где $Z^{d}$ - множество ячеек, $W$

* Department of Mathematical Sciences, Tsinghua University, Beijing, China 
- множество состояний частицы в каждой ячейке, которое в данной статье предполагается конечным. Каждая система определяется семейством вероятностных "скоростей" $\left\{c_{T}(\eta, d \xi), \eta \in E\right\}$, с которыми система переходит из одного состояния в другое. Здесь индексом $T$ помечено конечное подмножество из $Z^{d}$, и $c_{T}(\eta, d \xi)$ есть положительная мера на $W^{T}$ для каждого $\eta \in E$. Точное описание эволюции системы дано в гл. I книги [1]. Мы примем следуюшее

ПРЕДПОЛОЖЕнИЕ. Каждая рассматриваемая система $\left\{c_{T}(\eta, d \xi), \eta \in E\right\}$ удовлетворяет условиям теоремы 4.6 из гл. I книги [1]. Именно, предполагается, что

$$
\sup _{i} \sum_{T: i \in T}|T| c_{T}<\infty
$$

$u$

$$
\sup _{i} \sum_{T: i \in T} \sum_{j \neq i} c_{T}(j)<\infty, \quad \sup _{j} \sum_{i} \sum_{T: i \in T} c_{T}(j)<\infty
$$

әде

$$
c_{T} \equiv \sup _{\eta} c_{T}\left(\eta, W^{T}\right)
$$

$u$

$$
c_{T}(j) \equiv \sup \left\{\left\|c_{T}\left(\eta_{1}, d \zeta\right)-c_{T}\left(\eta_{2}, d \zeta\right)\right\|_{T}: \eta_{1}(k)=\eta_{2}(k) \forall k \neq j\right\}
$$

$a\|\cdot\|_{T}$ обозначает норму полной вариачии для обобщенной мерь на $W^{T}$.

Важным следствием этого предположения является то, что семейство $\left\{c_{T}(\eta, d \xi), \eta \in\right.$ $E\}$ определяет единственный непрерывный однородный марковский процесс $\left\{P_{\eta}, \eta \in E\right\}$ с траекториями из пространства $\Omega=D([0, \infty), E)$ функций $[0, \infty) \mapsto E$, непрерывных справа и имеющих предел слева в каждой точке $t \in[0, \infty)$. Соответствующая марковская полугруппа обозначается символом $\mathbf{S}=\{S(t), t \geqslant 0\}$, а семейство $\mathbf{S}$-инвариантных вероятностных мер - символом $m_{i}(E)$. Обозначим через $m_{s}(E)$ совокупность трансляционно-инвариантных вероятностных мер на $E^{1)}$.

Система называется эргодической, если $m_{i}(E)=\{\nu\}$ (одноэлементное множество) и для любого начального вероятностного распределения $\mu$ закон распределения $\mu S(t)$ при $t \rightarrow \infty$ стремится к $\nu$ в слабом смысле. Для многих интересных трансляционно-инвариантных систем единственность $\mathbf{S}$-инвариантной меры следует из единственности трансляционно-инвариантной $\mathbf{S}$-инвариантной меры (см., например, [1], гл. III). Поэтому необходимо исследовать различные свойства мер из множества $m_{i}(E) \cap m_{s}(E)$. Как отмечалось выше, одним из важных свойств является эргодичность относительно сдвигов.

Мера $\nu \in m_{s}(E)$ называется эргодической относительно сдвигов, если $\forall f, g \in C_{b}(E)$

$$
\lim _{n \rightarrow \infty} \frac{1}{\left|\Lambda_{n}\right|} \sum_{i \in \Lambda_{n}} \int f\left(\theta_{i} \cdot\right) g d \nu=\nu(f) \nu(g)
$$

\footnotetext{
${ }^{1)}$ Имеются в виду трансляции в $Z^{d}$.
} 
где $\Lambda_{n}=\left\{i \in Z^{d}:-n<i_{j} \leqslant n, 1 \leqslant j \leqslant d\right\},\left|\Lambda_{n}\right|$ - число элементов в $\Lambda_{n}, \theta_{i}$ - оператор сдвигов в $Z^{d}$. Известно, что это определение эквивалентно следующему: каждая инвариантная относительно пространственных сдвигов функция, заданная на $E$ и измеримая, $\nu$-почти всюду совпадает с постоянной.

Мера $\nu \in m_{s}(E)$ называется перемешивающей, если $\forall f, g \in C_{b}(E)$

$$
\lim _{i \rightarrow \infty} \int f\left(\theta_{i} \cdot\right) g d \nu=\nu(f) \nu(g)
$$

где $i=\left(i_{1}, \ldots, i_{d}\right) \rightarrow \infty$ означает, что $i_{j} \rightarrow \infty$ при $1 \leqslant j \leqslant d$. Если обозначить множество крайних мер из $m_{i}(E)$ символом $m_{i}^{e}(E)$ и множество крайних мер из $m_{i}(E) \cap m_{s}(E)$ символом $\left(m_{i}(E) \cap m_{s}(E)\right)_{e}$, то вопросы, заданные в работе [2], можно сформулировать следующим образом:

1. Является ли каждая мера в $\left(m_{i}(E) \cap m_{s}(E)\right)_{e}$ эргодической при сдвигах?

2. Является ли каждая мера в $m_{i}^{e}(E) \cap m_{s}(E)$ перемешивающей?

В данной работе мы даем утвердительные ответы на эти вопросы для класса систем взаимодействуюших частищ, включаюшего следуюшие системы.

1. Притягивающиеся системыпереориентируюшихся спинов. Для таких систем $W=$ $\{0,1\}, c_{T}(\cdot, \cdot)=0$ при $|T| \neq 1$. Если $T=\{i\}$, то $c_{T}(\eta,\{1-\eta(i)\})$ обозначается символом $c(i, \eta)$ и называется скоростью переориентации спина в ячейке $i$ (см. [1], гл. III). Система переориентирующихся спинов называется притягивающейся, если для любых двух конфигураций $\eta, \xi \in E$ с $\eta(j) \leqslant \xi(j) \forall j \in Z^{d}$ мы имеем

$$
\begin{aligned}
& c(i, \eta) \leqslant c(i, \xi), \text { если } \eta(i)=\xi(i)=0, \\
& c(i, \eta) \geqslant c(i, \xi), \text { если } \eta(i)=\xi(i)=1 .
\end{aligned}
$$

Такие системы вызывают особый интерес. Большинство детально исследованных систем переориентирующихся спинов, таких как ферромагнитная стохастическая модель Изинга, система избирателей, процессы контактов и т.п., являются притягивающимися. Важным свойством этих систем является существование нижней и верхней инвариантных мер, причем обе такие меры эргодичны относительно сдвигов, а система эргодична тогда и только тогда, когда эти меры совпадают.

2. Системы переориентируюшихся спинов со скоростями переориентации спинов, определенными формулами (4.3) или (4.4) в книге [1] (гл. III, § 4):

$$
c(i, \eta)=c(i)\left\{1-\eta(i)+[2 \eta(i)-1] \sum_{A \in \mathbf{Y}} p(i, A) H_{1}(i, \eta)\right\}
$$

или

$$
c(i, \eta)=\frac{c(i)}{2}\left\{1-[2 \eta(i)-1] \sum_{A \in \mathbf{Y}} p(i, A) H_{2}(i, \eta)\right\},
$$


где $Y$ - множество конечных подмножеств $Z^{d} \cup\{\Delta\}, \Delta$ - произвольный элемент, не принадлежащий $Z^{d}, c(i) \geqslant 0, p(i, A) \geqslant 0, \sup _{i} c(i)<\infty$ и

$$
\sup _{i} \sum_{A \in \mathbf{Y}} p(i, A) \leqslant 1, \quad \sup _{i} c(i) \sum_{A} p(i, A)|A|<\infty .
$$

$H_{i}$ задаются выражениями

$$
\begin{aligned}
& H_{1}(i, \eta)= \begin{cases}\prod_{i \in A \cap Z^{d}}[1-\eta(i)], & \Delta \notin A, \\
-\prod_{i \in A \cap Z^{d}}[1-\eta(i)], & \Delta \in A ;\end{cases} \\
& H_{2}(i, \eta)= \begin{cases}\prod_{i \in A \cap Z^{d}}[2 \eta(i)-1], & \Delta \notin A, \\
-\prod_{i \in A \cap Z^{d}}[2 \eta(i)-1], & \Delta \in A .\end{cases}
\end{aligned}
$$

3. Системы с исключением. Для таких систем $W=\{0,1\}$. Если $T=\{i, j\}, i \neq j$, то

$$
c_{T}(\eta,\{(\eta(j), \eta(i))\})=p(i, j),
$$

и $c_{T}(\cdot, \cdot)=0$ в остальных случаях, т.е. состояния ячеек могут только меняться местами. Числа $p(\cdot, \cdot)$ образуют матрицу переходных вероятностей некоторой марковской цепи с траекториями в $Z^{d}$. Процесс исключений называется симметричным, если $p(i, j)=$ $p(j, i) \forall i, j$.

4. Системы избирателей с тремя возможными стохастически предвзятыми мнениями. Для таких систем множество $W=\{1,2,3\}$ состоит из номеров трех мнений, одно из которых может иметь каждый избиратель, $c_{T}(\cdot, \cdot)=0$, если $|T| \neq 1$, и

$$
\begin{aligned}
& c_{\{i\}}(\eta,\{k\})=\alpha \sum_{j \in i+\mathbf{N}} I_{\{\eta(j)=k\}}+\delta_{k}, \quad k=1,2, \\
& c_{\{i\}}(\eta,\{3\})=\lambda \sum_{j \in i+\mathbf{N}} I_{\{\eta(j)=3\}}+\delta_{3},
\end{aligned}
$$

где $0 \leqslant \alpha \leqslant \lambda$, т.е. мнение 3 предпочтительнее двух других, $\mathbf{N}$ - множество ближайших к началу отсчета ячеек, $\delta_{k} \geqslant 0$.

5. Система избирателей со многими возможными мнениями. В такой системе $W$ является произвольным конечным множеством, $c_{T}(\cdot, \cdot)=0$, если $|T| \neq 1$, и

$$
c_{\{i\}}(\eta,\{k\})=\delta_{k}+\sum_{j \in i+\mathbf{N}} I_{\{\eta(j)=k\}}, \quad k \in W .
$$

Основное упрощение при исследовании таких систем состоит в том, что с каждой из них ассоциирован дуальный процесс - марковская цепь с непрерывным временем. Такие процессы просты и детально исследованы.

В разделе 2 мы доказываем общие теоремы, позволяющие с помощью дуальности установить эргодичность относительно сдвигов и перемешивание. В разделе 3 эти теоремы применяются к вышеописанным системам взаимодействуюших частиц. 


\section{2. ОБШИЕ РЕЗУЛЬТАТЫ}

В этом разделе мы исследуем проблемы эргодичности в обшем случае.

Пусть $E=W^{Z^{d}}$, где $W$ - польское пространство, $\left\{P_{\eta}, \eta \in E\right\}$ - однородный марковский процесс с непрерывным временем и траекториями в пространстве $\Omega=D([0, \infty), E)$, $\mathbf{S}=\{S(t), t \geqslant 0\}$ - соответствуюшая полугруппа. Семейства $m_{i}(E), m_{s}(E)$ определены в разделе 1. Справедливо следуюшее утверждение.

ТЕОРема 2.1. 1. Пусть $\nu \in\left(m_{i}(E) \cap m_{s}(E)\right)_{e}$ и для любъх ограниченных функиий f $и$ н на $E$

$$
\lim _{i \rightarrow \infty} \int\left|S(t)\left(f_{i} g\right)-S(t) f_{i} S(t) g\right| d \nu=0
$$

где $f_{i}=f\left(\theta_{i} \cdot\right)$. Если существует последовательность $t_{n} \rightarrow+\infty$ такая, что для каждой функиии $f$ из плотного подмножсества в $C_{b}(E)$ существует измеримая

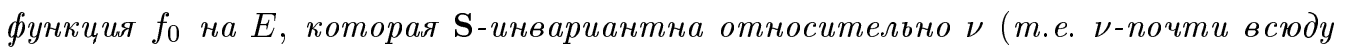
$\left.S(t) f_{0}=f_{0} \quad \forall t \geqslant 0\right)$ и такова, что

$$
\lim _{n \rightarrow \infty} S\left(t_{n}\right) f=f_{0} \quad \nu \text {-почти всюду }
$$

то мера $\nu$ әргодична относительно сдвигов.

2. Пусть $\nu \in m_{i}^{e}(E) \cap m_{s}(E)$ и справедливо равенство (2). Если для кажсдой функиии $f$ из некоторого плотного подмножества в $C_{b}(E)$ существует последовательность $t_{n} \rightarrow \infty$ и измеримая функиия $f_{0}$, являющаяся $\mathbf{S}$-инвариантной от-

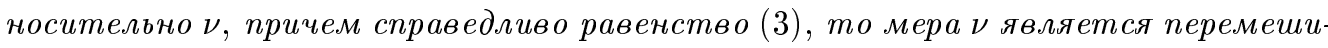
вающей.

В частности, если прочесс эргодичен (т.е. множество $m_{i}(E)=\{\nu\}$ состоит из одного әлемента и для любой начальной конфигурации $\eta$ вероятностное распределение $P_{\eta}\left(\omega_{t} \in \cdot\right)$ прочесса в момент $t$ сходится $\kappa \nu$ при $\left.t \rightarrow \infty\right)$, то мера $\nu$ является перемешивающей.

Чтобы доказать теорему 2.1, мы воспользуемся нижеследующими утверждениями об эргодичности при пространственно-временных сдвигах и экстремальностью инвариантных мер (теорема 2.2 и леммы 2.1 и 2.2 ). В результате получим способ устанавливать единственность элементов в $m_{i}(E) \cap m_{s}(E)$.

ЛЕмма 2.1 ([3], лемма 5.4.24). Пусть $\Phi$ - аффинная функиия, действующая из

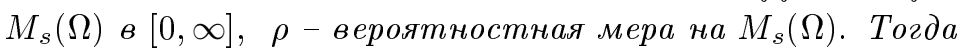

$$
\Phi\left(\int Q \rho(d Q)\right)=\int \Phi(Q) \rho(d Q) .
$$


TеOрема 2.2. Пусть $\nu \in m_{i}(E) \cap m_{s}(E) u$

$$
P_{\nu}=\int P_{\eta} \nu(d \eta)
$$

Тогда существует единственная вероятностная мера $\rho$ на множсестве $M_{s, s}^{e}(\Omega)$ эргодических при пространственно-временных сдвигах вероятностных мер на $\Omega=$ $D([0, \infty), E)$ такая, что

$$
P_{\nu}=\int Q \rho(d Q)
$$

и р-почти все прочессь $Q$ являются марковскими на $\Omega$ с полугруппой $S(t)$, т.е. $Q=\int P_{\eta} \mu_{Q}(d \eta)$, где $\mu_{Q}$ является одновременнбім маргинальньм распределением для $Q . \quad$ В частности, $\nu \in\left(m_{i}(E) \cap m_{s}(E)\right)_{e}$ тогда и только тогда, когда мера $P_{\nu}$ является әргодической при пространственно-временных сдвигах. Более того, любъе две различные меры в $\left(m_{i}(E) \cap m_{s}(E)\right)_{e}$ взаимно сингулярны.

ДокАЗАтЕльСтво. Мы используем энтропию Донскера-Варадхана $H$ на $M_{s}(\Omega)$ для марковских процессов, где $M_{s}(\Omega)$ - пространство стационарных вероятностных мер на $\Omega$. Определение и свойства энтропии $H$ изложены в книге [4]. Здесь мы используем только следующее свойство:

Энтропия $H$ является аффинной и полунепрерьвной снизу на $M_{s}(\Omega)$. Если $Q$ является марковской на $\Omega$ с полугруппой $\{S(t), t \geqslant 0\}$, то $H(Q)=0$.

Пусть равенство (4) - эргодичное при пространственно-временных сдвигах разложение меры $P_{\nu}$ (оно единственно). Тогда из приведенного выше свойства и леммы 2.1 следует, что

$$
0=H\left(P_{\nu}\right)=\int H(Q) \rho(d Q) .
$$

Используя вторую часть приведенного свойства, мы устанавливаем, что $\rho$-почти все $Q$ являются марковскими на $\Omega$ с полугруппой $S(t)$. Тогда для $\rho$-почти всех $Q$ имеем $\nu_{Q} \in$ $m_{i}(E) \cap m_{s}(E)$ и

$$
\nu=\int \nu_{Q} \rho(d Q)
$$

Отсюда следует, что $\nu \in\left(m_{i}(E) \cap m_{s}(E)\right)_{e}$ тогда и только тогда, когда $P_{\nu} \in M_{s, s}^{e}(\Omega)$.

Для доказательства взаимной сингулярности мер заметим, что для каждой меры $\nu \in\left(m_{i}(E) \cap m_{s}(E)\right)_{e}$ и ограниченной измеримой функции $f$ на $E$ согласно известной эргодической теореме имеем

$$
\lim _{n \rightarrow \infty} \frac{1}{n\left|\Lambda_{n}\right|} \sum_{i \in \Lambda_{n}} \int_{0}^{n} f\left(\theta_{i} \omega_{t}\right) d t=\nu(f) \quad P_{\nu} \text {-почти всюду и по мере } P_{\nu} .
$$

Отсюда непосредственно следует, что $\forall f \in C_{b}(E)$

$$
\lim _{n \rightarrow \infty} \frac{1}{n\left|\Lambda_{n}\right|} \sum_{i \in \Lambda_{n}} \int_{0}^{n} S(t) f\left(\theta_{i} \cdot\right) d t=\nu(f) \quad \nu \text {-почти всюду. }
$$

Это показывает, что любые две различные меры в $\left(m_{i}(E) \cap m_{s}(E)\right)_{e}$ взаимно сингулярны. 


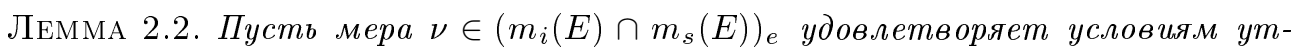
верждения 1 теоремы 2.1. Тогда для каждой ограниченной измеримой функиии $f$ на Е существует подпоследовательность последовательности $\left\{t_{n}\right\}$ и измеримая функиия $f_{0}$, которая S-инвариантна относительно $\nu$, такая, что справедливо равенство (3). Более того, если функция $f$ инвариантна относительно пространственных сдвигов, то $f_{0}=\nu(f)$ р-почти всюду.

ДокАЗАТЕльство. Доказательство первого утверждения стандартно. Достаточно только аппроксимировать $f$ функциями из $C_{b}(E)$ в топологии $L^{2}(\nu)$ и использовать сингулярность $\nu$.

Докажем второе утверждение. Допустим, что функция $f$ инвариантна относительно пространственных сдвигов. Тогда легко проверить, что функция $f_{0}$ тоже инвариантна относительно пространственных сдвигов. По теореме 2.2 из условия $\nu \in\left(m_{i}(E) \cap\right.$ $\left.m_{s}(E)\right)_{e}$ следует, что мера $P_{\nu}$ эргодична относительно пространственно-временных сдвигов. Поэтому

$$
\begin{aligned}
\int\left|f_{0}-\nu(f)\right| d \nu & =\int\left|\frac{1}{(2 n)^{d} n} \sum_{i \in \Lambda_{n}} \int_{0}^{n} E^{P_{\eta}} f_{0}\left(\left(\theta_{t, i} \omega\right)_{0}\right) d t-\int f_{0}\left(\omega_{0}\right) d P \nu\right| d \nu \leqslant \\
& \leqslant \int\left|\frac{1}{(2 n)^{d} n} \sum_{i \in \Lambda_{n}} \int_{0}^{n} f_{0}\left(\left(\theta_{t, i} \omega\right)_{0}\right) d t-\int f_{0}\left(\omega_{0}\right) d P_{\nu}\right| d P_{\nu} \rightarrow 0
\end{aligned}
$$

при $n \rightarrow \infty$, где $\Lambda_{n}=Z^{d} \cap[-n, n)^{d}, \theta_{t, i}$ - оператор пространственно-временного сдвига на $\Omega_{+}$. Отсюда непосредственно следует второе утверждение леммы.

ДоКАЗАТЕЛЬСТВО ТЕОРЕМЫ 2.1. 1. Пусть $g$-неотрицательная ограниченная измеримая пространственно-инвариантная функция на $E$. Для произвольного $\epsilon>0$ выберем ограниченную функцию $f$ на $E$ такую, что $\int|f-g| d \nu<\epsilon$. Тогда $\forall i \in Z^{d}$

$$
\int f_{i} f d \nu-\epsilon(\|f\|+\|g\|) \leqslant \int g^{2} d \nu=\int g_{i} g d \nu \leqslant \int f_{i} f d \nu+\epsilon(\|f\|+\|g\|)
$$

Здесь и далее $\|g\|=\sup _{\eta \in E}|g(\eta)|$. Согласно лемме 2.2 можно выбрать последовательность $t_{n} \rightarrow \infty$ такую, что $S\left(t_{n}\right) g \rightarrow \nu(g) \nu$-почти всюду. Для каждого $n \geqslant 1$ имеем

$$
\left|\int f_{i} f d \nu-[\nu(f)]^{2}\right| \leqslant \Delta_{i, n}^{1}+\Delta_{i, n}^{2}
$$

где

$$
\begin{aligned}
& \Delta_{i, n}^{1}=\left|\int S\left(t_{n}\right) f_{i}\left[S\left(t_{n}\right) f-\nu(f)\right] d \nu\right|, \\
& \Delta_{i, n}^{2}=\int\left|S\left(t_{n}\right)\left(f_{i} f\right)-S\left(t_{n}\right) f_{i} S\left(t_{n}\right) f\right| d \nu .
\end{aligned}
$$


Для заданного $\epsilon>0$ в силу выбора $f$ имеем

$$
\Delta_{i, n}^{1} \leqslant \epsilon(\|f\|+\|g\|)+\|f\| \int\left|S\left(t_{n}\right) g-\nu(g)\right| d \nu .
$$

Поэтому существует номер $n$ такой, что $\Delta_{i, n}^{2} \leqslant \epsilon(2\|f\|+\|g\|) \forall i$. Фиксируя $t_{n}$ и перейдя к пределу при $i \rightarrow \infty$, получим $\Delta_{i, n}^{2} \rightarrow 0$. Итак,

$$
\lim _{i \rightarrow \infty} \sup \left|\int f_{i} f d \nu-[\nu(f)]^{2}\right| \leqslant c \epsilon
$$

для некоторой постоянной $c>0$. Из (5) следует, что

$$
\left|\int g^{2} d \nu-[\nu(g)]^{2}\right| \leqslant 2 \epsilon(\|f\|+\|g\|)+\lim _{i \rightarrow \infty} \sup \left|\int f_{i} f d \nu-[\nu(f)]^{2}\right| .
$$

Таким образом, $\operatorname{Var}_{\nu}(g)=0$, т.е. $g=$ const $\nu$-почти всюду. Следовательно, мера $\nu$ эргодична относительно сдвигов.

2. Пусть $f$ - ограниченная функция на $E$ и $g \in C_{b}(E)$. Выберем последовательность $t_{n} \rightarrow \infty$ и измеримую функцию $g_{0}$, которая $\mathbf{S}$-инвариантна относительно $\nu$, такие, что $S\left(t_{n}\right) g \rightarrow g_{0} \nu$-почти всюду при $n \rightarrow \infty$. Используя экстремальность $\nu$, нетрудно проверить, что $g_{0}=\nu(g) \nu$-почти всюду. Итак, для каждого $n \geqslant 1$

$$
\begin{aligned}
\left|\int f_{i} g d \nu-\nu(f) \nu(g)\right| \leqslant & \int\left|S\left(t_{n}\right)\left(f_{i} g\right)-S\left(t_{n}\right) f_{i} S\left(t_{n}\right) g\right| d \nu+ \\
& +\left|\int S\left(t_{n}\right) f_{i}\left[S\left(t_{n}\right) g-\nu(g)\right] d \nu\right| .
\end{aligned}
$$

Теперь рассуждения, аналогичные использованным при доказательстве утверждения 1 , приводят к равенству

$$
\lim _{i \rightarrow \infty} \int f_{i} g d \nu=\nu(f) \nu(g) .
$$

ЗАмЕчАниЕ. Для проверки условий теоремы 2.1 сначала следует убедиться в том, что для каждого $T>0$ и каждой $f$ из некоторого плотного подмножества в $C_{b}(E)$ справедливо равенство

$$
\lim _{t \rightarrow \infty}|S(t+T) f(\eta)-S(t) f(\eta)|=0 \quad \forall \eta \in E .
$$

Затем нужно доказать, что для каждой функции $f$ в этом плотном подмножестве из $C_{b}(E)$ можно найти последовательность $t_{n}$ такую, что $S\left(t_{n}\right) f$ сходится поточечно на $E$. Тогда из (5) следует, что предельная функция является $\mathbf{S}$-инвариантной, т.е. что $S(t) f=f$ всюду на $E \forall t \geqslant 0$. В следующем разделе будет показано, что равенство (6) справедливо для любого устойчивого марковского процесса с непрерывным временем. Используя этот факт и дуальность аналогично тому, как это будет сделано при доказательстве следствия (см. ниже), мы можем выполнить эти два шага для систем взаимодействуюших частиц, описанных в разделе 1 . В частности, равенство (6) справедливо для любой такой системы. Заметим, что равенство (6) представляет самостоятельный интерес. 
СледСтвиЕ. Пусть справедливо равенство (2) и существует дуальный марковский процесс на некотором вероятностном пространстве с пространством состояний $\widehat{E}$ и полугруппой $\{\widehat{S}(t), t \geqslant 0\}$ такой, что

1) для каждой ограниченной измеримой функции $\hat{f}$ на $\widehat{E}$ и любой последовательности $t_{n} \rightarrow \infty$ существует подпоследовательность $t_{n_{k}}$ такая, ито предель

$$
\lim _{k \rightarrow \infty} \widehat{S}\left(t_{n_{k}}\right) \hat{f}=\hat{f}_{0}
$$

существуют и функция $\hat{f}_{0}$ является $\widehat{\mathbf{S}}$-инвариантной;

2) для каждой функции $f$ на $E$ существует конечный набор ограниченных измеримых функиий $\hat{f}_{1}, \ldots, \hat{f}_{k}$ на $E \times \widehat{E}$ таких, что для каждого $\eta \in E$ существуют точки $x_{1}, \ldots, x_{k} \in \widehat{E}$, для которых

$$
S(t) f(\eta)=\sum_{i=1}^{k} \widehat{S}(t) \hat{f}_{i}(\eta, \cdot)\left(x_{i}\right) \quad \forall t \geqslant 0 .
$$

Тогда справедливы все утверждения теоремы 2.1 .

ДокАЗАтЕльСтво. Заметим, что множество функций на $E$ счетно. Следовательно, множество соответствующих дуальных функций, фигурирующих в условии 2 тоже счетно. Совместно с условием 1 это показывает, что выполнены условия утверждения 1 теоремы 2.1.

Теорему 2.1 можно применять и к обратимым системам.

Теорема 2.3. Пусть полугруппа $\mathbf{S}$ обратима относительно некоторой меры $\nu \in m_{i}(E)$ и справедливо равенство (2). Если $\nu \in\left(m_{i}(E) \cap m_{s}(E)\right)_{e}$, то мера $\nu$ әргодична относительно сдвигов. Если $\nu \in m_{i}^{e}(E) \cap m_{s}(E)$, то $\nu$ - перемешивающая мера.

ДОКАЗАТЕЛЬСТво. Поскольку полугруппа $\mathbf{S}$ обратима относительно $\nu$, то для каждой функции $f \in C_{b}(E)$ предел

$$
f_{0}=\lim _{t \rightarrow \infty} S(t) f
$$

сушествует $\nu$-почти всюду, и функция $f_{0} \mathrm{~S}$-инвариантна относительно $\nu$. Поэтому утверждение теоремы следует из теоремы 2.1.

\section{3. ПРИМЕНЕНИЕ РЕЗУЛЬТАТОВ РАЗДЕЛА 2 К СИСТЕМАМ ВЗАИМОДЕЙСТВУЮШИХ ЧАСТИЦ}

В силу предположения, сформулированного в разделе 1 , для рассматриваемых систем взаимодействуюших частиц имеем

$$
\lim _{i \rightarrow \infty} \sup _{0 \leqslant t \leqslant T}\left\|S(t)\left(f_{i} g\right)-S(t) f_{i} S(t) g\right\|=0
$$

для любого $T>0$ и для любых функций $f$ и $g$ на $E$. Поэтому равенство (2) справедливо для любой меры $\nu$. Следовательно, остается реализовать шаги, намеченные в замечании (см. раздел 2), используя дуальность. Мы ограничимся рассмотрением одного класса систем взаимодействуюших частиц, охватывающего системы, описанные в разделе 1. 
ТЕОРема 3.1. Пусть для системь взаимодействующих частии $\left\{c_{T}(\eta, d \xi)\right.$, $\eta \in E\}$ справедливо равенство (7). Предположим, что

1) существует устойчивый марковский процесс с непрерывным временем $\left\{\widehat{P}_{A}\right.$, $A \in \widehat{E}\}$ с некоторым счетныц пространством состояний $\widehat{E}$ и полугруппой $\widehat{\mathbf{S}}=$ $\{\widehat{S}(t), \quad t \geqslant 0\}$

2) существует ограниченная измеримая функиия $H(\cdot, \cdot)$ на $E \times \widehat{E}$ такая, что линейнье комбинации $H(\cdot, A)$ как функиии на $E$ плотнь в $C_{b}(E)$;

3) дуальное соотношение

$$
S(t) H(\cdot, A)(\eta)=\widehat{E}^{A} H\left(\eta, A_{t}\right) \exp \left\{-\int_{0}^{t} V\left(A_{u}\right) d u\right\} \quad \forall(\eta, A) \in E \times \widehat{E} \quad u \quad t \geqslant 0
$$

въполняется для некоторой неотрицательной измеримой функции $V$ на $\widehat{E}$.

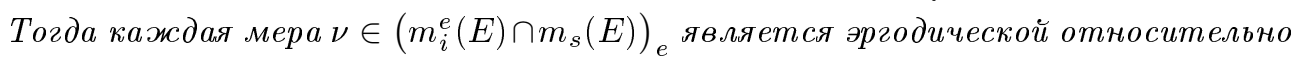
сдвигов и каждая мера $\nu \in m_{i}^{e}(E) \cap m_{s}(E)$ является перемешивающей. В частности,

$$
\left(m_{i}^{e}(E) \cap m_{s}(E)\right)_{e}=m_{i}(E) \cap m_{s}^{e}(E),
$$

где $m_{s}^{e}(E)$ - множество мер в $m_{s}(E)$, әргодических относительно сдвигов.

Мы докажем эту теорему в два этапа, указанных в замечании в разделе 2 , т.е. мы должны сначала проверить равенство (6). Это сводится к доказательству следуюшей леммы.

Лемма 3.1. Пусть $\mathbf{S}=\{S(t), t \geqslant 0\}-$ устойчивый марковский прочесс с непрерывными временем и пространством состояний Е. Тогда для каждой ограниченной измеримой функции $f$ на $E$ и любого $T>0$ равенство (6) справедливо на Е. Более того, если $V$ - неотрицательная измеримая функиия на $E$, то $\forall x \in E$

$$
\lim _{t \rightarrow \infty}\left|E^{x} f\left(X_{t+T}\right) \exp \left\{-\int_{0}^{t+T} V\left(X_{u}\right) d u\right\}-E^{x} f\left(X_{t}\right) \exp \left\{-\int_{0}^{t} V\left(X_{u}\right) d u\right\}\right|=0 .
$$

ЛЕмма 3.2 ([5], гл. $6, \S 6$, теорема 1.7). Пусть $p_{n}(\cdot, \cdot)$ - переходные вероятности $n$-го порядка некоторой марковской чепи с пространством состояний Е. Положим

$$
\alpha_{k}=\sup _{x} \lim _{n \rightarrow \infty}\left\|p_{n+k}(x, \cdot)-p_{n}(x, \cdot)\right\|,
$$

где $\|\cdot\|$ означает норму полной вариации для обобщенной меры на $E$. Тогда $\alpha_{k}=0$ или 2. 
ДокАЗАТЕЛЬСТво лЕмМЫ 3.1. Для любого $x \in E$ определим функцию

$$
\phi(t)=S(t+T) f(x)-S(t) f(x) .
$$

Тогда, поскольку $\{S(n T), n \geqslant 0\}$ является марковской цепью на $E$ и

$$
|S((n+1) T) g(x)-S(n T) g(x)| \leqslant 1-e^{T q_{x}}<1
$$

для любой измеримой функции $g$ на $E$ с $\|g\| \leqslant 1$ и любого $x \in E$ с $q_{x}>0$, где $q_{x}$-интенсивность скачков процесса, начинающегося в точке $x$, из леммы 3.2 следует, что

$$
\lim _{n \rightarrow \infty} \phi(n T)=0
$$

Фиксируем произвольное $T>0$. Поскольку функшия $S(t) f(x)$ равномерно-непрерьвна по $t$, для каждого $\epsilon>0$ существует число $\delta>0$ такое, что

$$
\left|S\left(t_{1}\right) f(x)-S\left(t_{2}\right) f(x)\right|<\epsilon
$$

при $\left|t_{1}-t_{2}\right|<\delta$. Пусть $0<T_{1}<\delta$. Тогда $n T_{1}=N_{n} T+\sigma_{n} T$ при $N_{n} \in \mathbb{Z}_{+}$и $0 \leqslant \sigma_{n}<1$. Поскольку $S(t)$ - марковская полугруппа на счетном пространстве $E$, легко проверить, что справедливо неравенство

$$
\begin{aligned}
& \left|S\left(n T_{1}+T\right) f(x)-S\left(n T_{1}\right) f(x)\right|=\left|S\left(N_{n} T+T+\sigma_{n} T\right) f(x)-S\left(N_{n} T+\sigma_{n} T\right) f(x)\right| \leqslant \\
& \leqslant e^{T q_{x}} \sum_{y}\left|S\left(\left(N_{n}+1\right) T\right) f(y)-S\left(N_{n} T\right) f(y)\right| p(T, x,\{y\}),
\end{aligned}
$$

где $p(t, x, A)$ - переходные вероятности для процесса $S(t)$. Используя теорему Лебега о мажорированной сходимости, имеем

$$
\lim _{n \rightarrow \infty}\left|S\left(n T_{1}+T\right) f(x)-S\left(n T_{1}\right) f(x)\right|=0 .
$$

Для большого $t>0$ выберем номер $n \geqslant 1$ такой, что $n T_{1} \leqslant t<(n+1) T_{1}$. Тогда

$$
|S(t+T) f(x)-S(t) f(x)| \leqslant\left|S\left(n T_{1}+T\right) f(x)-S\left(n T_{1}\right) f(x)\right|+2 \epsilon .
$$

Комбинируя это неравенство с соотношением (9) и учитывая произвольность $\epsilon$, получаем

$$
\lim _{t \rightarrow \infty} \phi(t)=0
$$

что доказывает первое утверждение леммы. 
Чтобы доказать второе утверждение леммы, заметим, что для любого $t_{0}>0$ справедливо неравенство

$$
\begin{aligned}
& \left|E^{x} f\left(X_{t+T}\right) \exp \left\{-\int_{0}^{t+T} V\left(X_{u}\right) d u\right\}-E^{x} f\left(X_{t}\right) \exp \left\{-\int_{0}^{t} V\left(X_{u}\right) d u\right\}\right| \leqslant \\
& \leqslant \Delta_{T}^{1}(t)+\Delta_{T}^{2}(t)+\Delta_{T}^{3}(t),
\end{aligned}
$$

где

$$
\begin{aligned}
& \Delta_{T}^{1}(t)=\left|E^{x}\left[f\left(X_{t+T}\right)-f\left(X_{t}\right)\right]\left[\exp \left\{-\int_{0}^{t+T} V\left(X_{u}\right) d u\right\}-\exp \left\{-\int_{0}^{t_{0}} V\left(X_{u}\right) d u\right\}\right]\right| \\
& \Delta_{T}^{2}(t)=\left|E^{x}\left[f\left(X_{t+T}\right)-f\left(X_{t}\right)\right] \exp \left\{-\int_{0}^{t_{0}} V\left(X_{u}\right) d u\right\}\right| \\
& \Delta_{T}^{3}(t)=\left|E^{x} f\left(X_{t}\right)\left[\exp \left\{-\int_{0}^{t+T} V\left(X_{u}\right) d u\right\}-\exp \left\{-\int_{0}^{t} V\left(X_{u}\right) d u\right\}\right]\right|
\end{aligned}
$$

Нетрудно проверить, что для любого $\epsilon>0$ и достаточно больших $t_{0}>0$ и $t>t_{0}$ имеем $\Delta_{T}^{1}(t)<\epsilon$. В силу марковского свойства и первого утверждения леммы получаем

$$
\lim _{t \rightarrow \infty} \Delta_{T}^{2}(t)=\left|\lim _{t \rightarrow \infty} E^{x} \exp \left\{-\int_{0}^{t_{0}} V\left(X_{u}\right) d u\right\} E^{X_{t_{0}}}\left[f\left(X_{t-t_{0}+T}\right)-f\left(X_{t-t_{0}}\right)\right]\right|=0
$$

и, как нетрудно проверить,

$$
\lim _{t \rightarrow \infty} \Delta_{T}^{3}(t)=0
$$

Отсюда следует второе утверждение леммы.

ДОКАЗАТЕЛЬСТВО ТЕОРЕМЫ 3.1. ИспольЗуя лемму 3.1, легко показать, что при выполнении условий теоремы для каждой функции $f \in C_{b}(E)$ равенство (6) справедливо на $E$. Более того, если $f \in C_{b}(E)$ - линейная комбинация функций $H\left(\cdot, A_{i}\right)$ для некоторых $A_{i}, 1 \leqslant i \leqslant k$, то

$$
S(t) f(\eta)=\sum_{i=1}^{k} \widehat{E}^{A_{i}} H\left(\eta, A_{t}\right) \exp \left\{-\int_{0}^{t} V\left(X_{u}\right) d u\right\}
$$

Поскольку $\widehat{E}$ - счетное множество, используя диагональный процесс, можно найти последовательность $t_{n} \rightarrow \infty$ такую, что поточечный предел

$$
f_{0}=\lim _{n \rightarrow \infty} S\left(t_{n}\right) f
$$

сушествует для такой функции $f$ и функция $f_{0}$ является $\mathbf{S}$-инвариантной в силу равенства (6). Поскольку по предположению семейство всех таких линейных комбинаций плотно в $C_{b}(E)$, то утверждения теоремы следуют из теоремы 2.1 . 
Применяя полученные результаты к системам, описанным в разделе 1 , приходим к следующим выводам:

1. Для систем переориентируюшихся притягиваюшихся спинов с взаимодействием конечного радиуса, которые удовлетворяют условиям, приведенным в работе [6], справедливы утверж дения теоремы 3.1. В частности, нижняя и верхняя инвариантные меры являются перемешивающими.

2. Для систем переориентируюшихся спинов со скоростями переориентации, определенными формулами (1a), (1б), и для симметричных процессов исключения из гл. III и VIII книги [1] известно, что справедливы утверждения теоремы 3.1.

3. Для системы избирателей с тремя возможными стохастически предвзятыми мнениями и для систем избирателей со многими возможными мнениями из результатов работы [7] нетрудно вывести, что условия теоремы 3.1 выполнены. Следовательно, для таких систем справедливы утверждения теоремы 3.1 .

4. Теорема 2.3 применима к мере Гиббса, ассоциированной с любой стохастической моделью Изинга, удовлетворяющей равенству (7), например с моделью Изинга с взаимодействием конечного радиуса (не обязательно притягиваюшим).

Благодарности. Работа выполнена при поддержке NSF Китая.

\section{Список литературы}

[1] T. M. Liggett. Interacting Particle Systems. New York: Springer, 1985.

[2] E. D. Andjel. Trans. Am. Math. Soc. 1990. V. 318. P. 601-614.

[3] J. D. Deuschel, D. W. Stroock. Large Deviations. Boston, MA: Academic Press, 1989.

[4] S. R. S. Varadhan. Large Deviations and Applications. Philadelphia: SIAM, 1984.

[5] D. Revuz. Markov Chains. Amsterdam: North-Holland, 1984.

[6] M. Katori. J. Phys. A. 1994. V. 27. P. 3191-3211.

[7] F. J. López, G. Sanz. Markov Process. Related Fields. 2000. V. 6. № 3. P. 305-328.

Поступила в редакцию 24.VIII.2000 г., после доработки 23.VIII.2001 г. 